$$
\overline{\mathbf{p}}(\boldsymbol{\xi})=-\frac{\sqrt[3]{\pi p}}{2 a} \cdot e^{-\xi^{2 a 2}},
$$

so that

$$
\sigma_{x}=-\frac{a p}{\sqrt{\pi}} \int_{0}^{\infty} e^{-\xi^{2} a^{2}} \cos (\xi y) d \xi=-\frac{p}{2} e^{-y^{2 /\left(4 a^{2}\right)}}
$$

\section{Acknowledgment}

The author wishes to express his hearty thanks to Manager T. Kameoka and Mr. E. Sato for their valuable suggestions.

\section{References}

(1) cf. L.N.G. Filon: Phil. Trans. Roy. Soc. Lond., Ser. A, Vol. 201 (1903), p. 63.

(2) I.N. Sneddon: Proc. Cambridge Phil.Soc., Vol. 42, (1946), p. 260 .

(3) M. Hannah: Quart. Jour. Mech. Appl. Math., Vol. N, Part 1 (1951).

(4) I.N.Sneddon: Fourier Transforms, (1951), McGraw Hill.

(5) B. An: Trans. Japan Soc. Mech. Engrs., Vol. 28, No. 185 (1962), p. 1.

$620.171 .5: 539.384 .2 .07$

\title{
Photoelastic Study of Flat Bars with Two Notches Subjected to Pure Bending*
}

\author{
By Yasunori Murakami** and Toshio KawABE***
}

\begin{abstract}
Flat bar specimens of Epoxy Resin with two U notches of identical shape on one edge were subjected to pure bending. The effects of the pitch of two notches, the notch depth and also the root radius on the stress concentration factors were investigated. As to the effect of the pitch, the stress concentration factor of two notches decreased at first owing to the overlapping effect, and then showed an increase up to the value of a single notch. With respect to the effects of the notch depth and the root radius, the stress concentration factor of two notches showed a similar change to that of a single notch. In all cases examined, the stress concentration factors of two notches obtained were smaller than those of a single notch. This fact offers a possibility of reducing the stress concentration due to a single notch by a supplementary notch formed in its neighbourhood.
\end{abstract}

\section{Introduction}

The authors have carried out photoelastic studies on flat bars with a single notch and with a single double notch under pure bending. ${ }^{(1)}$ An extensive photoelastic study on flat bars with a single notch under pure bending is reported by Nishida. ${ }^{(2)}$ In this experiment, as a preliminary to the case of multiple notches, which is encountered, for example, in screw threads, the case of two notches was investigated. In the case of multiple notches, in genieral, the overlapping effect appears, and the stress concentration factor is supposed to take a smaller value than that of a single notch. Neuber studied theoretically the case of round bars

* Received 8 th May, 1961

** Professor, Faculty of Science and Engineering, Ritsumeikan University, Kita-ku, Kyoto.

*** Assistant, Faculty of Science and Engineering, Ritsumeikan University. with multiple circumferential grooves subjected to torsion, and derived a stress-reducing factor (Entlastungszahl). ${ }^{(3)}$ Hirano conducted a theoretical analysis on the relief of stress concentration for the case of flat bars with multiple notches under tension. ${ }^{(4)}$ Durelli et al examined photoelastically the effect of the pitch on the stress concentration factors of flat bars with multiple semi-circular notches subjected to tension. ${ }^{(5)}$ Recently, Okubo proposed new formulas for the reduction of stress concentration for the case of multiple notches and circumferential grooves. ${ }^{(6)}$ In all cases abovementioned, the overlapping effect appeared so as to decrease the stress concentration factor, and this fact is of vital importance in the design of notched members.

In this experiment, the stress concentration factors of flat bars with two $U$ notches of identical shape formed on one edge were studied under pure 
bending, and the effects of the pitch of two notches, the notch depth and also the root radius on the stress concentration factors were examined. Also, the experimental results were compared with those of a single notch, and a stress concentration reduction factor (which may be designated as such) was calculated for each case.

\section{Experimental procedures}

\subsection{Specimens}

Specimens used were of Epoxy Resin plate with a thickness of $3 \mathrm{~mm}$, and the shape of the specimen is illustrated in Fig. 1. The width of the specimen $b=20 \mathrm{~mm}$ was kept constant, and the pitch $p$, the notch depth $t$ and the root radius $\rho$ were varied in several steps. Specimens were finished by hand, with special caution to avoid dimensional errors and to prevent edge stresses. Dimensions of specimens after hand finishing were measured by a universal projector with $\times 20$ magnification. Measured dimensions are summarized in Table 1, and the maximum deviation from a predetermined dimension is confined to about $1 \%$. Photoelastic investigation was carried out immediately after finishing in order to avoid time-edge effect. Loading apparatus used was the same as the one in the previous experiment ${ }^{(1)}$.

\subsection{Calculation of the stress concentration factors}

Formerly, the determination of the fringe order at the notch root was conducted by an extrapolation method, in which fringe order distribution curves were drawn from fringe patterns. This method has the defect of introducing some errors into the maximum fringe orders to be determined through the extrapolation of distribution curves, and also a considerable trouble is required for the determination of the final fringe orders. Accordingly, in this study, to remove these imperfections, Tardy's methcd was employed. This method consists in adjusting the darkest part of one fringe to the edge of the notch by rotating the polarizer. This procedure is quite easy, and the fraction of the fringe orders can be calculated from the angle of rotation of the polarizer. Fig. 2 shows some examples of fringe patterns for two notches. Bending moments applied to the specimens were varied in four to six steps, and the moment versus fringe order curves were drawn. These curves should be straight lines passing through the origin, and in case that these lines deviated from the origin, correction lines were drawn parallel to the original lines, since these deviations might be attributed to the effect of the initial or edge stresses. One example of the correction lines is illustrated in Fig. 3. To examine the reliability of this new method, the fringe orders determined in the above way were compared,

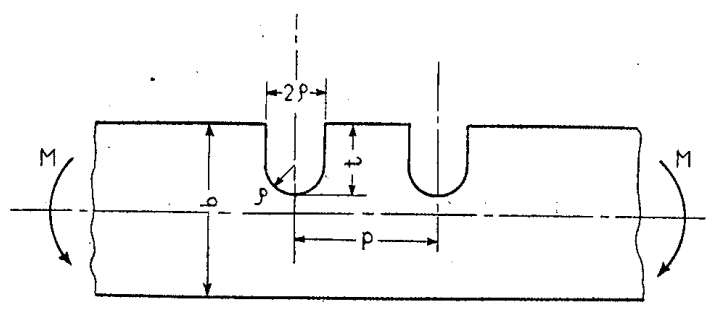

Fig. 1 Shape of specimen

Table 1 Dimensions of specimens

\begin{tabular}{|c|c|c|c|c|c|c|c|c|c|c|c|}
\hline \multicolumn{3}{|c|}{ Specimen number } & 1 & 2 & 3 & 4 & 5 & 6 & 7 & 8 & 9 \\
\hline \multirow{6}{*}{ 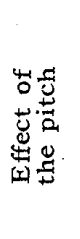 } & \multirow{3}{*}{$\begin{array}{c}\rho / b=0.15 \\
t / b=0.3\end{array}$} & $p$ & 7.97 & 10.20 & 13.86 & 15.73 & 21.99 & 27.97 & 39.81 & - & - \\
\hline & & $b-t$ & 13.93 & 14.09 & 13.99 & 14.26 & 14.15 & 14.07 & 14.00 & - & - \\
\hline & & $p / 2 \rho$ & 1.335 & 1.675 & 2.238 & 2.616 & 3.620 & 4.685 & 6.730 & - & - \\
\hline & \multirow{3}{*}{$\begin{array}{l}\rho / b=0.15 \\
t / b=0.5\end{array}$} & $p$ & 6.10 & 7.93 & 9.85 & 12.00 & 16.00 & 17.99 & 22.17 & 27.98 & 40.12 \\
\hline & & $b-t$ & 9.96 & 9.98 & 10.05 & 10.00 & 9.80 & 9.95 & 9.92 & 9.93 & 10.09 \\
\hline & & $p / 2 \rho$ & 0.993 & 1.290 & 1.660 & 1.890 & 2.640 & 2.900 & 3.650 & 4.680 & 6.730 \\
\hline \multirow{6}{*}{ 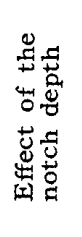 } & \multirow{3}{*}{$\begin{aligned} f / b & =0.15 \\
p / 2 \rho & =1.33\end{aligned}$} & $t$ & 1.04 & 2.06 & .3 .23 & 3.97 & 4.97 & 6.01 & 10.04 & 12.07 & - \\
\hline & & $b-t$ & 18.98 & 17.98 & 16.79 & 16.05 & 15.07 & 14.01 & 10.00 & 7.95 & - \\
\hline & & $t / b$ & 0.052 & 0.103 & 0.161 & 0.199 & 0.248 & 0.300 & 0.501 & 0.603 & - \\
\hline & \multirow{3}{*}{$\begin{aligned} \rho / b & =0.15 \\
p / 2 \rho & =2.67\end{aligned}$} & $t$ & 1.01 & 2.04 & 3.10 & 3.98 & 6.08 & 8.04 & 10.20 & 12.07 & - \\
\hline & & $b-t$ & 18.97 & 17.98 & 16.89 & 16.03 & 14.19 & 11.95 & 9.80 & 7.94 & - \\
\hline & & $t / b$ & 0.051 & 0.102 & 0.155 & 0.199 & 0.300 & 0.402 & 0.510 & 0.600 & - \\
\hline \multirow{6}{*}{ 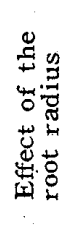 } & \multirow{3}{*}{$\begin{array}{c}t / b=0.3 \\
p / 2 \rho=1.33\end{array}$} & $\rho$ & 1.98 & 2.97 & 6.05 & 11.96 & 20.00 & - & - & - & - \\
\hline & & $b-t$ & 14.00 & 13.92 & 14.04 & 14.07 & 14.02 & - & - & - & - \\
\hline & & $\rho / b$ & 0.099 & 0.149 & 0.302 & 0.598 & 1.000 & - & - & - & - \\
\hline & \multirow{3}{*}{$\begin{array}{c}t / b=0.3 \\
p / 2 p=2.67\end{array}$} & $\rho$ & 1.97 & 3.01 & 6.04 & 12.00 & 19.95 & - & - & - & - \\
\hline & & $b-t$ & 14.03 & 14.26 & 14.01 & 13.95 & 14.03 & - & - & - & - \\
\hline & & $\rho / b$ & 0.098 & 0.150 & 0.302 & 0.600 & 0.996 & - & - & - & - \\
\hline
\end{tabular}




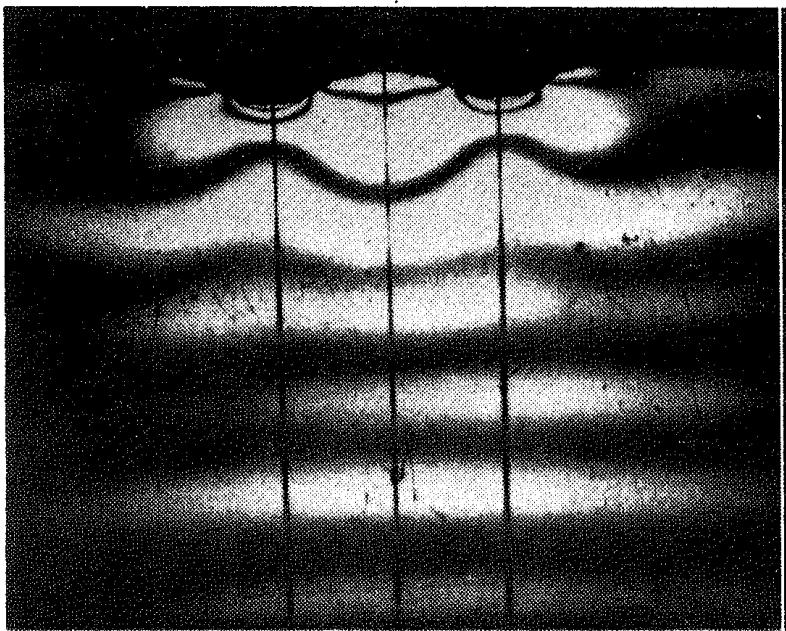

$\rho / b=0.15$

$p / 2 \rho=1.33$

$t / b=0.052$

$M=19.52 \mathrm{~kg} \mathrm{~cm}$

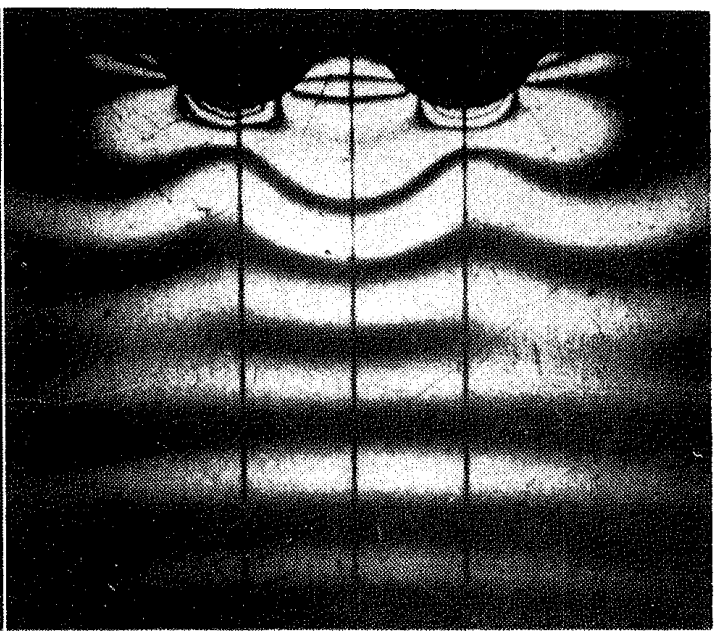

$\rho / b=0.15$

$p / 2 \rho=1.33$

$t / b=0.103$

$M=18.56 \mathrm{~kg} \mathrm{~cm}$

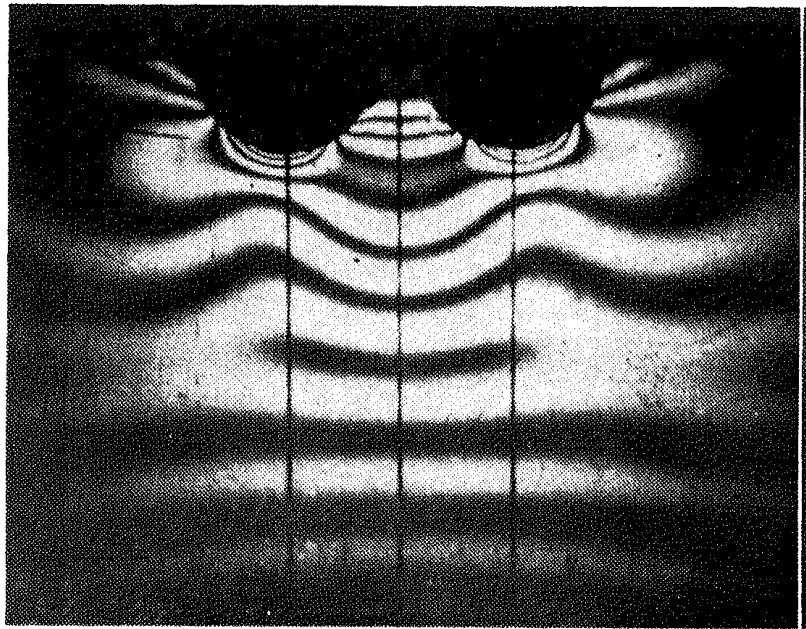

$\rho / b=0.15$

$p / 2 \rho=1.33$

$t / b=0.161$

$M=18.56 \mathrm{~kg} \mathrm{~cm}$

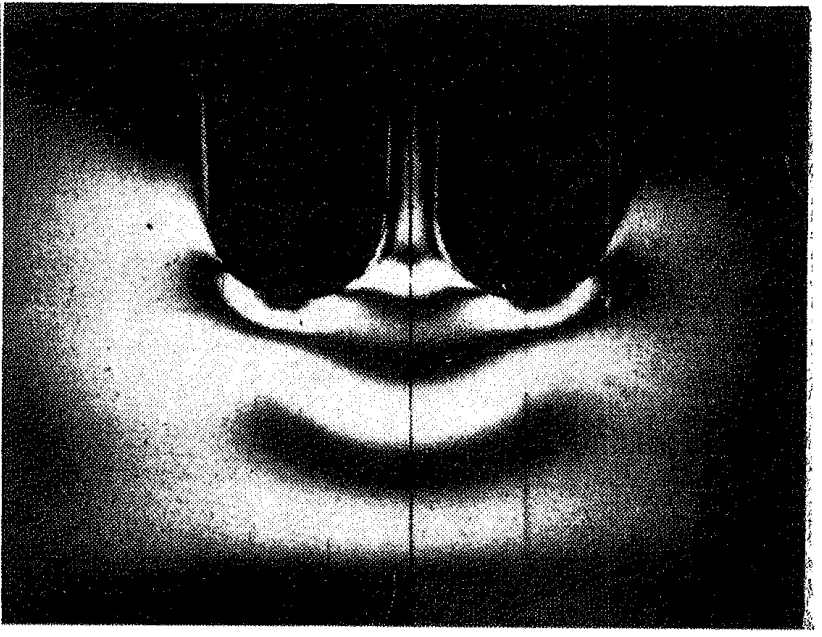

$\rho / b=0.15$

$p / 2 \rho=1.33$

$t / b=0.501$

$M=3.68 \mathrm{~kg} \mathrm{~cm}$

Fig. 2 Examples of fringe patterns

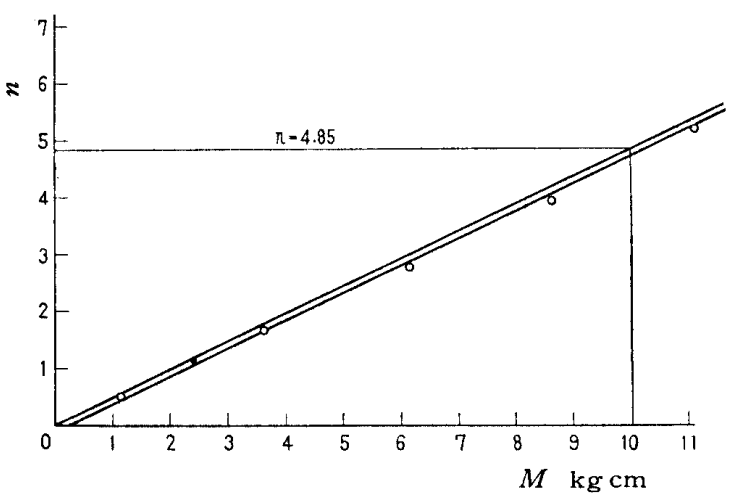

Fig. 3 One example of correction lines (Tardy's method)

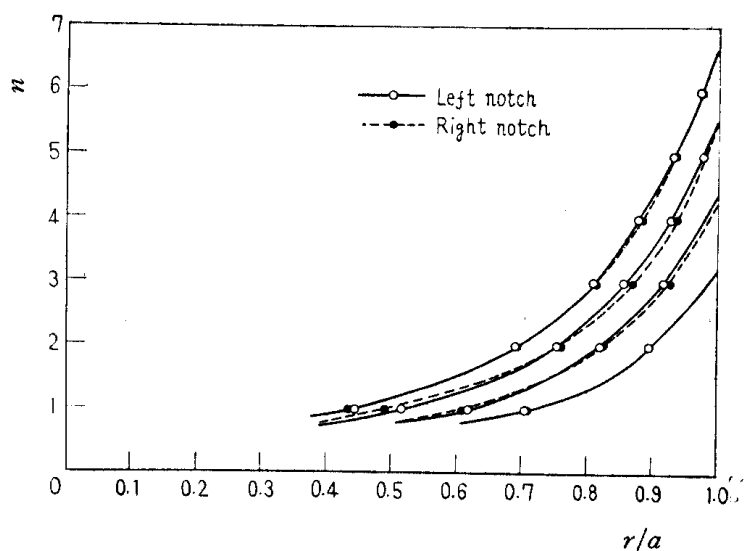

Fig. 4 One example of fringe order curves (extrapolation method) 
in some cases of the whole experiment, with those obtained by the prior extrapolation method, and the maximum deviation was confined to less than $1 \%$, showing a satisfactory agreement. This is demonstrated in Figs. 4 and 5, which show the fringe order curve and the correction line, respectively, according to the extrapolation method applied to the same specimen as that of Fig. 3. The final fringe orders determined by both methods are quite the same in this instance.

Next, in this experiment, the maximum fringe order appeared at a point on the notch contour which was located slightly outside of the notch root. The line connecting this point and the center of the semi-circle of the notch root forms a deviation angle $\theta$. This phenomenon was not observed in the case of a single notch, and this may be due to the interference of two notches. In this experiment, fringe patterns were sketched and from these patterns deviation angles were measured. Stress concentration factors were calculated, as usual, by the following formula:

$$
\alpha=\frac{n / \sigma}{n_{0} / \sigma_{0}}
$$

where $n$ and $\sigma$ are the maximum fringe order and the nominal bending stress at the point where the maximum fringe order appeared, and $n_{0}, \sigma_{0}$ the maximum fringe order and the maximum bending stress for a control specimen, respectively.

\section{Results of experiment}

\subsection{The effect of the pitch}

Experiment was carried out for the two cases of $t=6 \mathrm{~mm}(t / b=0.3)$ and $t=10 \mathrm{~mm}(t / b=0.5)$, the root radius $\rho=3 \mathrm{~mm}(\rho / b=0.15)$ being kept constant and the pitch of two notches being varied in several steps. The results are shown in Table 2 and Fig. 6. In Fig. 6, open circles show the stress concentration factors of a single notch, which are plotted from the previous experiment. The stress concentration factors of two notches are indicated by semi-solid and solid circles for the cases of $t / b=0.3$ and 0.5 , respectively. As is apparent in the figure, the stress concentration factor of two notches is less than that of a single notch in the range of small values of $p / 2 \rho$. It shows a minimum at a certain value of $p / 2 \rho$, and thereafter increases, approaching finally to that of a single notch. The amount of $p / 2 \rho$ at which the stress concentration factor takes a minimum value is larger for a deeper notch, and in Fig. 6 this amount is $p / 2 \rho \fallingdotseq 1.7$ and $p / 2 \rho \doteqdot 2.5$ for the cases of $t / b=0.3$ and 0.5 , respectively. The value of $p / 2 \rho$ at which the stress concentration factor of two notches comes

Table 2 Results of experiment (Effect of the pitch)

\begin{tabular}{|c|c|c|c|c|c|c|c|c|c|c|c|}
\hline 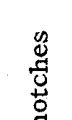 & $\begin{array}{c}\rho / b=0.15 \\
t / b=0.3\end{array}$ & $\begin{array}{c}p / 2 \rho \\
\boldsymbol{\alpha} \\
\boldsymbol{\theta}^{\circ}\end{array}$ & $\begin{array}{r}1.335 \\
1.60 \\
8.0\end{array}$ & $\begin{array}{r}1.675 \\
1.58 \\
7.5\end{array}$ & $\begin{array}{r}2.238 \\
1.64 \\
6.3\end{array}$ & $\begin{array}{r}2.616 \\
1.75 \\
5.0\end{array}$ & $\begin{array}{r}3.620 \\
1.77 \\
0\end{array}$ & $\begin{array}{r}4.685 \\
1.76 \\
0\end{array}$ & $\begin{array}{r}6.730 \\
1.77 \\
0\end{array}$ & $\begin{array}{l}- \\
- \\
-\end{array}$ & - \\
\hline$\stackrel{\circ}{H}$ & $\begin{array}{l}\rho / b=0.15 \\
t / b=0.5\end{array}$ & $\begin{array}{c}p / 2 \rho \\
\boldsymbol{\alpha} \\
\boldsymbol{\theta}^{\circ}\end{array}$ & $\begin{array}{r}0.993 \\
1.46 \\
8.0\end{array}$ & $\begin{array}{r}1.290 \\
1.45 \\
8.5\end{array}$ & $\begin{array}{r}1.660 \\
1.42 \\
8.0\end{array}$ & $\begin{array}{r}1.890 \\
1.40 \\
7.5\end{array}$ & $\begin{array}{r}2.640 \\
1.40 \\
6.0\end{array}$ & $\begin{array}{r}2.900 \\
1.42 \\
5.1\end{array}$ & $\begin{array}{r}3.650 \\
1.49 \\
2.8\end{array}$ & $\begin{array}{r}4.680 \\
1.55 \\
0\end{array}$ & $\begin{array}{r}6.730 \\
1.55 \\
0\end{array}$ \\
\hline 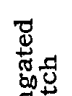 & $\begin{array}{c}\rho / b=0.15 \\
t / b=0.3\end{array}$ & $\begin{array}{r}p / 2 \rho \\
\boldsymbol{\alpha} \\
\end{array}$ & $\begin{array}{r}1.34 \\
1.53 \\
\end{array}$ & $\begin{array}{r}1.68 \\
1.48 \\
\end{array}$ & $\begin{array}{l}2.24 \\
1.49 \\
\end{array}$ & $\begin{array}{r}2.62 \\
1.53 \\
\end{array}$ & $\begin{array}{l}3.62 \\
1.64\end{array}$ & $\begin{array}{l}4.69 \\
1.63\end{array}$ & $\begin{array}{l}6.73 \\
1.47\end{array}$ & - & - \\
\hline 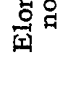 & $\begin{array}{l}\rho / b=0.15 \\
t / b=0.5\end{array}$ & $\begin{array}{r}p / 2 \rho \\
\boldsymbol{\alpha}\end{array}$ & $\begin{array}{l}1.29 \\
1.35\end{array}$ & $\begin{array}{l}1.66 \\
1.31\end{array}$ & $\begin{array}{l}1.89 \\
1.31\end{array}$ & $\begin{array}{l}2.64 \\
1.29\end{array}$ & $\begin{array}{l}2.90 \\
1.32\end{array}$ & $\begin{array}{l}3.65 \\
1.38\end{array}$ & $\begin{array}{l}4.68 \\
1.41\end{array}$ & $\begin{array}{l}6.73 \\
1.24\end{array}$ & - \\
\hline
\end{tabular}

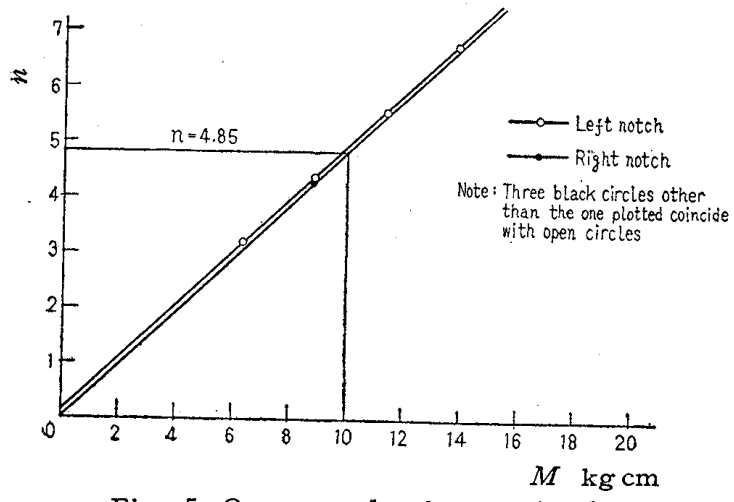

Fig. 5 One example of correction lines (extrapolation method)

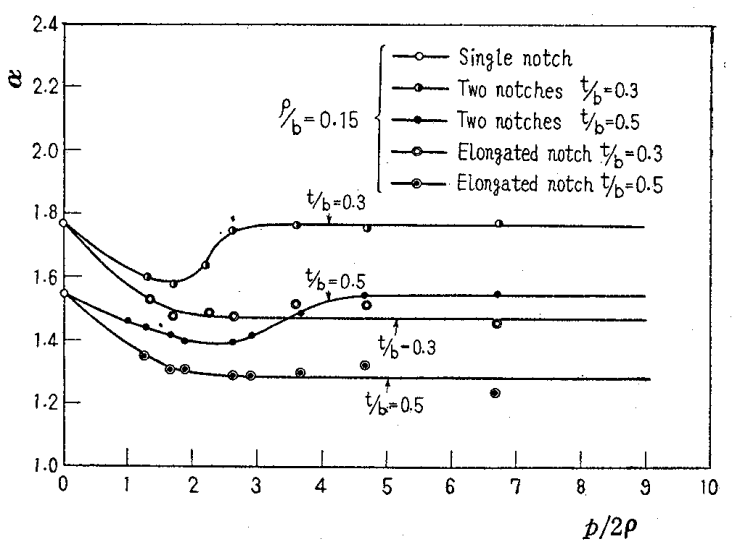

Fig. 6 Effect of the pitch on the stress concentration factors 
back to that of a single notch as a result of the disappearance of the overlapping effect is, from Fig. $6, \quad p / 2 \rho \fallingdotseq 3$ and $p / 2 \rho \doteqdot 5$ for the cases of $t / b=$ 0.3 and 0.5 , respectively. From this, it is understood that the region of the appearance of the overlapping effect is wider for a deeper notch. This tendency of the change in the stress concentration factors is in agreement with the experimental results obtained by Durelli et al for flat bars with multiple semicircular notches subjected to tension ${ }^{(5)}$, and also with those obtained by the authors for flat bars with multiple circular holes under tension( ${ }^{(7)}$.

Next, the intermediate part between two notches was cut off, so as to form an elongated notch, and the stress concentration factor of this notch was examined. The results are indicated by double circles in Fig. 6 . In both cases of $t / b=0.3$ and 0.5 , the stress concentration factor decreases at first from that of a single notch, and then maintains a constant value respectively. This definite value must coincide with the stress concentration factor of a shouldered plate, and it agrees fairly well with the value obtained from Peterson's $\operatorname{graph}^{(8)}$ for a

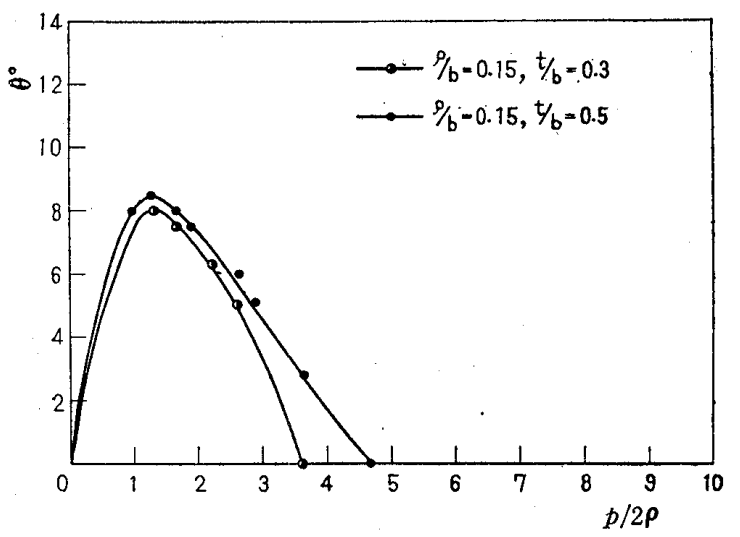

Fig. 7 Effect of the pitch on the deviation angles

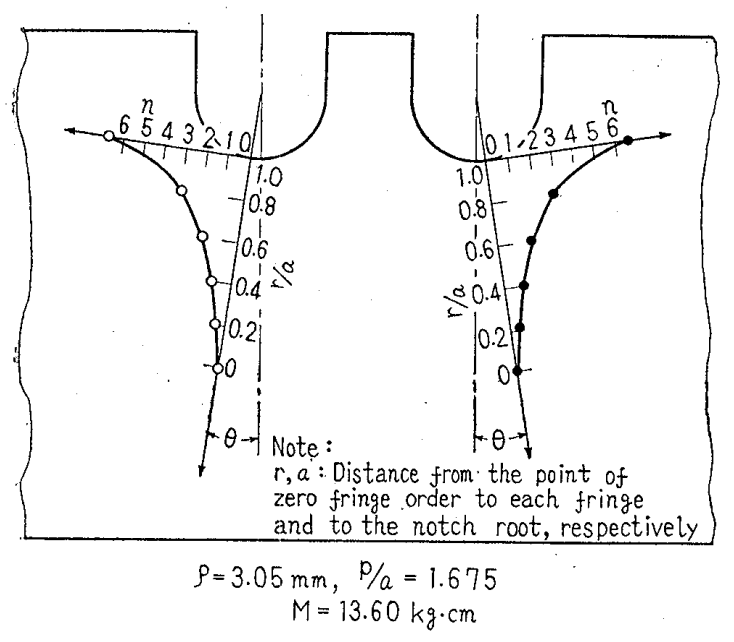

Fig. 8ne example of fringe order distribution curves shouldered plate with the double width of $2 b$. For the whole range of $p / 2 \rho$, the elongated notch shows a smaller stress concentration factor than two notches. This fact should be noted in design, which shows that it is more favorable to remove the intermediate part between two notches, in case that the stress concentration is to be reduced.

In the case of two notches, as mentioned in the paragraph $2 \cdot 2$, the maximum fringe order appears at the point on the notch configuration which is slightly deviated from the notch root, producing a deviation angle $\theta$. This angle was measured by a protractor in a $\times 20$ magnified sketch of the fringe pattern, and the result is shown in Fig. 7. In the figure, the deviation angle $\theta$ shows a peak value of nearly $8^{\circ}$ at $p / 2 \rho=1.3$ for both cases of $t / b=0.3$ and 0.5 , and thereafter decreases steeply. The range of pitch in which the deviation angle appears is in fairly good consistency with that in which the overlapping effect comes out. Fig. 8 shows one example of the fringe order distribution curves for two notches.

\subsection{The effect of the notch depth}

Experiment was made for the two cases of $p$ $=8 \mathrm{~mm}(p / 2 \rho=1.33)$ and $p=16 \mathrm{~mm}(p / 2 \rho=2.67)$, the root radius $\rho=3 \mathrm{~mm}(\rho / b=0.15)$ being kept constant. The results are summarized in Table 3 and Fig. 9. In the figure, for the sake of comparison, the result of the previous experiment on a single notch is shown in a chain line. Full lines indicate the results of two notches for the two cases of $p / 2 \rho=1.33$ (semi-solid circles) and 2.67 (solid circles). . The stress concentration factor $\alpha$ of two notches increases at first steeply, reaching a maximum value at about $t / b=0.1$ and 0.2 , and thereafter diminishes slowly to unity at $t / b=1.0$. This trend is quite similar to that of a single notch, but the value of $\alpha$ is smaller than that of a single notch in both cases. In the range of $t / b$ less ${ }_{i}^{7}$ than $0.45, \alpha$ takes a lower value for a smaller pitch. This is due to the overlapping effect, as is

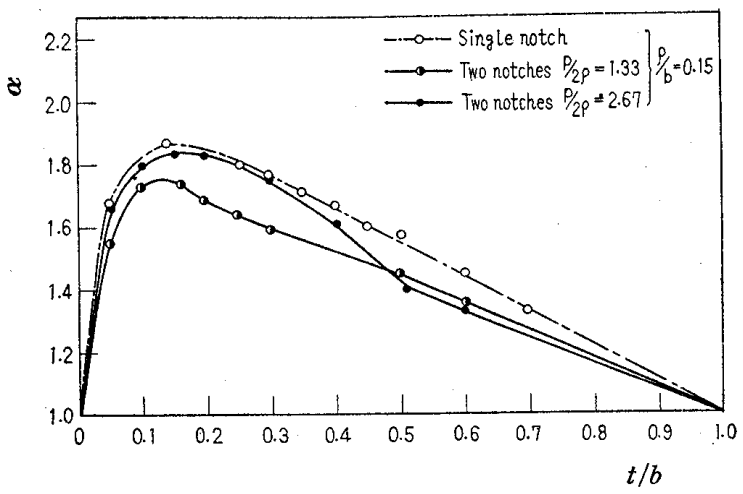

Fig. 9 Effect of the notch depth on the stress concentration factors 
Table 3 Results of experiment (Effect of the notch depth)

\begin{tabular}{|c|c|c|c|c|c|c|c|c|c|}
\hline $\begin{aligned} \rho / b & =0.15 \\
p / 2 \rho & =1.33\end{aligned}$ & $\begin{array}{l}t / b \\
\boldsymbol{\alpha} \\
\boldsymbol{\theta}^{\circ}\end{array}$ & $\begin{array}{r}0.052 \\
1.55 \\
1.3\end{array}$ & $\begin{array}{r}0.103 \\
1.73 \\
3.0\end{array}$ & $\begin{array}{r}0.161 \\
1.74 \\
6.0\end{array}$ & $\begin{array}{r}0.199 \\
1.69 \\
7.0\end{array}$ & $\begin{array}{r}0.248 \\
1.64 \\
7.5\end{array}$ & $\begin{array}{r}0.300 \\
1.59 \\
8.0\end{array}$ & $\begin{array}{r}0.501 \\
1.45 \\
8.5\end{array}$ & $\begin{array}{r}0.603 \\
1.36 \\
5.0\end{array}$ \\
\hline $\begin{aligned} \rho / b & =0.15 \\
p / 2 \rho & =2.67\end{aligned}$ & $\begin{array}{c}t / b \\
\boldsymbol{\alpha} \\
\boldsymbol{\theta}^{\circ}\end{array}$ & $\begin{array}{r}0.051 \\
1.66 \\
0\end{array}$ & $\begin{array}{r}0.102 \\
1.80 \\
1.0\end{array}$ & $\begin{array}{r}0.155 \\
1.84 \\
2.3\end{array}$ & $\begin{array}{r}0.199 \\
1.83 \\
3.5\end{array}$ & $\begin{array}{r}0.300 \\
1.75 \\
5.0\end{array}$ & $\begin{array}{r}0.402 \\
1.61 \\
8.0\end{array}$ & $\begin{array}{r}0.510 \\
1.40 \\
6.0\end{array}$ & $\begin{array}{r}0.600 \\
1.33 \\
0\end{array}$ \\
\hline
\end{tabular}

Table 4 Results of experiment (Effect of the root radius)

\begin{tabular}{c|c|r|r|r|r|r}
\hline \hline$t / b=0.3$ & $\rho / b$ & 0.099 & 0.149 & 0.302 & 0.598 & 1.000 \\
$p / 2 \rho=1.33$ & $\alpha$ & 1.85 & 1.60 & 1.41 & 1.27 & 1.13 \\
& $\theta^{\circ}$ & 8.0 & 8.0 & 6.7 & 4.2 & 4.0 \\
\hline \multirow{2}{*}{$t / b=0.3$} & $\rho / b$ & 0.098 & 0.150 & 0.302 & 0.600 & 0.996 \\
$p / 2 \rho=2.67$ & $\alpha$ & 2.08 & 1.75 & 1.51 & 1.32 & 1.17 \\
& $\theta^{\circ}$ & 4.8 & 5.0 & 3.8 & 2.2 & 1.8 \\
\hline
\end{tabular}

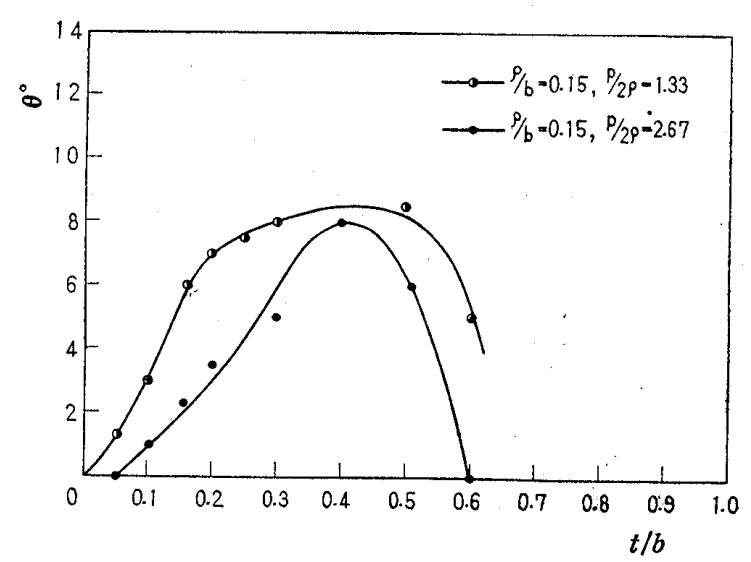

Fig. 10 Effect of the notch depth on the deviation angles

seen in Fig. 6. This situation becomes inverse for the range of $t / b$ larger than 0.45 , which results from the fact that the minimum value of $\dot{\alpha}$ shifts towards a larger pitch for a deeper notch. Moreover, the peak of $\alpha$ occurs at a deeper notch as the pitch increases. The deviation angle $\theta$ for this case is shown in Fig. 10. In this case also, $\theta$ shows a maximum value at about $t / b=0.4$. The range of $t / b$ in which $\theta$ appears is narrower for a larger pitch. In the case of $p / 2 \rho=2.67, \theta$ disappears at $t / b=0.6$, and in the case of $p / 2 \rho=1.33, \theta$ is supposed to vanish at about $t / b=0.7$ from the shape of the distribution curve.

\subsection{The effect of the root radius}

This effect was examined for the two cases of $p=8 \mathrm{~mm} \quad(p / 2 \rho=1.33)$ and $p=16 \mathrm{~mm}(p / 2 \rho=2.67)$, the notch depth $t / b=0.3$ being maintained constant. The results obtained are summarized in Table 4 and Fig. 11. In this figure also, the experimental result of a single notch is shown in a chain line. The value of $\alpha$ for two notches diminishes at first steeply, and then slowly with the increase in the root radius. Again, this tendency is quite similar to the case of a single notch, but two notches show

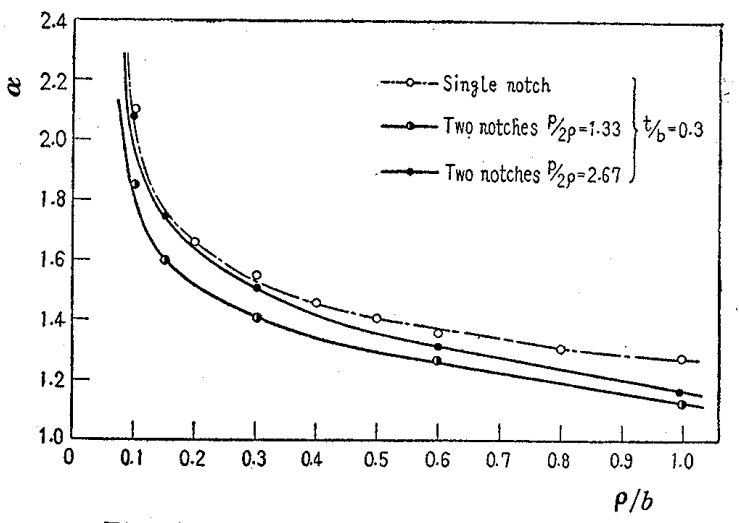

Fig. 11 Effect of the root radius on the stress concentration factors

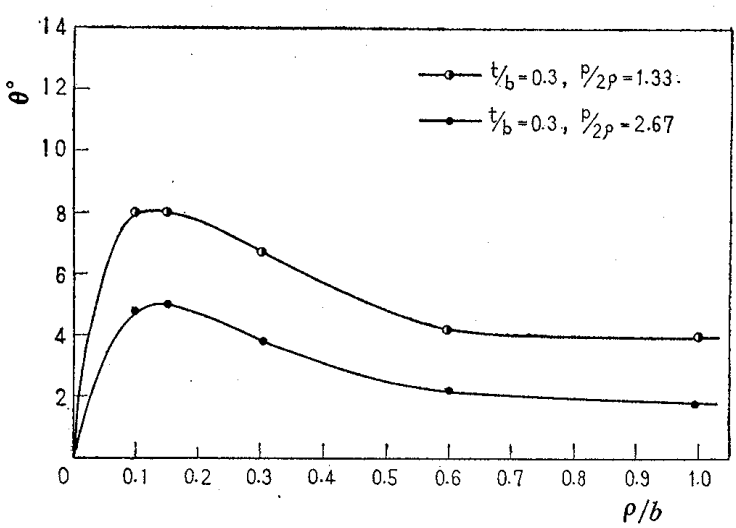

Fig. 12 Effect of the root radius on the deviation angles

smaller values of $\alpha$ in both cases of $p / 2 \rho=1.33$ and 2.67. This trend is especially conspicuous for the case of a smaller pitch. The change in the deviation angle for this case is shown in Fig. 12. These $\theta$-curves show respective peaks of $8^{\circ}$ and $5^{\circ}$ at about $\rho / b=0.1$. Dissimilar to the previous results of Figs. 7 and $9, \theta$ does not vanish for a finite value of $\rho / b$, having still the values of $4^{\circ}$ and $2^{\circ}$ at $\rho / b$ =1.0. $\theta$ is assumed to vanish for an infinite value of $\rho / b$.

3.4 Comparison of stress concentration factors between the two cases of a single notch and two notches

To obtain the relation between the stress concentration factors of a single notch and two notches, let $\alpha_{1}$ and $\alpha_{2}$ be the stress concentration factors of the above case respectively, and we put these in the following relation:

$\alpha_{2}=k \cdot \alpha_{1}$ 
The value of $k$ in the above equation can be calculated from the results of experiment. This coefficient $k$ may be designated as a stress concentration reduction factor, though it has a different meaning from the stress-reducing factor $\gamma$ in the equation of the effective depth $t_{m}=\gamma \cdot t$ derived by Neuber ${ }^{(3)}$. The values of $k$ obtained from the results shown in Figs. 6,9 and 11 are tabulated in Table 5, and are illustrated in Figs. 13, 14 and 15. Fig. 13 is the result for the effect of the pitch, and corresponds to Fig. 6 . These k-curves show similar shapes to the $\alpha$-curves in Fig. 6 , and the

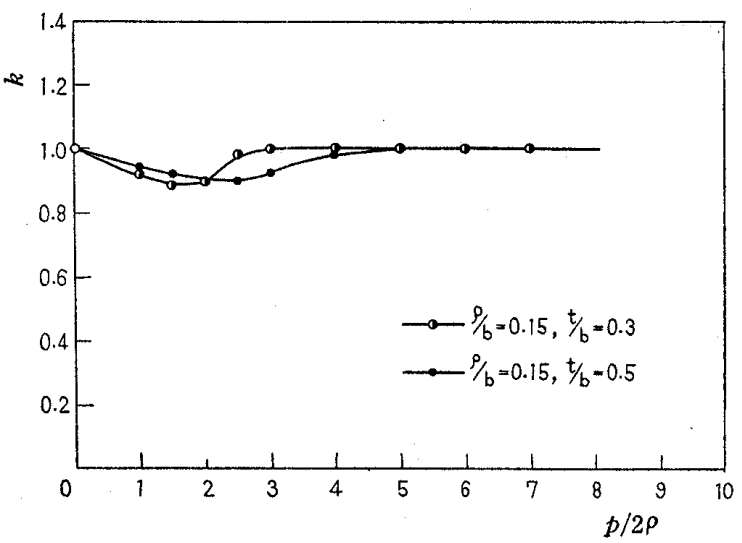

Fig. 13 Effect of the pitch on the $k$-values

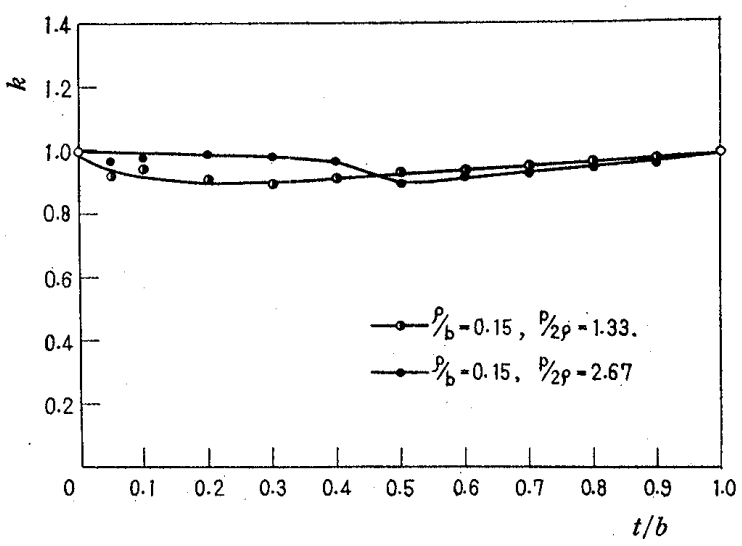

Fig. 14 Effect of the notch depth on the $k$-values value of $k$ is less than unity in the ranges of $p / 2 \rho$ $<3$ and $p / 2 o<5$ for the cases of $t / b=0.3$ and 0.5 , respectively. In other words, the range of $k<1$ corresponds to that in which the overlapping effect appears. For larger pitches than the abovementioned, the interference occurs no more between two notches, and $k$ takes the value of nearly unity. From these $k$-curves, the following experimental formulas are obtained:

$$
\begin{aligned}
& 1 \leqq p / 2 \rho \leqq 3(0.3 \leqq t / b \leqq 0.5) \\
& 0.1345(p / 2 \rho)^{2}-0.4075(p / 2 \rho)+1.163 \\
& \quad+\left[-0.235(p / 2 \rho)^{2}+0.645(p / 2 \rho)-0.310\right] \cdot t / b \\
& 3<p / 2 \rho \leqq 5(0.3 \leqq t / b \leqq 0.5) \\
& -0.0255(p / 2 \rho)^{2}+0.1275(p / 2 \rho)+0.988 \\
& \quad+\left[0.085(p / 2 \rho)^{2}-0.425(p / 2 \rho)+0.04\right] \cdot t / b
\end{aligned}
$$

Fig. 14 is the result for the effect of the notch depth, which corresponds to Fig. 9. As is seen in this figure, $k$-curves show an inverse tendency between the two cases of $p / 2 \rho=1.33$ and 2.67 for the separate ranges of $t / b$ bordered approximately by 0.45 . These results from the change in the value of $\alpha$ shown in Fig. 9, and $k$ become unity at $t / b=1.0$. The value of $k$ is less than unity for the whole range of $t / b$ except zero and unity in both cases. The experimental formulas are obtained

\begin{tabular}{|c|c|c|c|c|c|c|c|c|}
\hline \multicolumn{3}{|c|}{ Effect of the pitch } & \multicolumn{3}{|c|}{ Effect of the notch depth } & \multicolumn{3}{|c|}{ Effect of the root radius } \\
\hline$p / 2 \rho$ & $t / b=0.3$ & $t / b=0.5$ & $t / b$ & $p / 2 \rho=1.33$ & $p / 2 p=2.67$ & $\rho / b$ & $p / 2 \rho=1.33$ & $p / 2 \rho=2.67$ \\
\hline 1.0 & 0.92 & 0.94 & 0.1 & 0.95 & 0.98 & 0.1 & 0.89 & 0.97 \\
\hline 1.5 & 0.89 & 0.92 & 0.2 & 0.91 & 0.99 & 0.2 & 0.92 & 0.99 \\
\hline 2.0 & 0.90 & 0.90 & 0.3 & 0.90 & 0.98 & 0.3 & 0.92 & 0.99 \\
\hline 2.5 & 0.98 & 0.90 & 0.4 & 0.92 & 0.97 & 0.4 & 0.92 & 0.97 \\
\hline 3.0 & 1.00 & 0.92 & 0.5 & 0.94 & 0.90 & 0.5 & 0.92 & 0.96 \\
\hline 4.0 & 1.00 & 0.98 & 0.6 & 0.94 & 0.92 & 0.6 & 0.92 & 0.96 \\
\hline 5.0 & 1.00 & 1.00 & 0.7 & 0.95 & 0.93 & 0.7 & 0.92 & 0.96 \\
\hline 6.0 & 1.00 & 1.00 & 0.8 & 0.97 & 0.95 & 0.8 & 0.92 & 0.95 \\
\hline 7.0 & 1.00 & 1.00 & 0.9 & 0.98 & 0.97 & 0.9 & 0.90 & 0.94 \\
\hline
\end{tabular}
as follows:

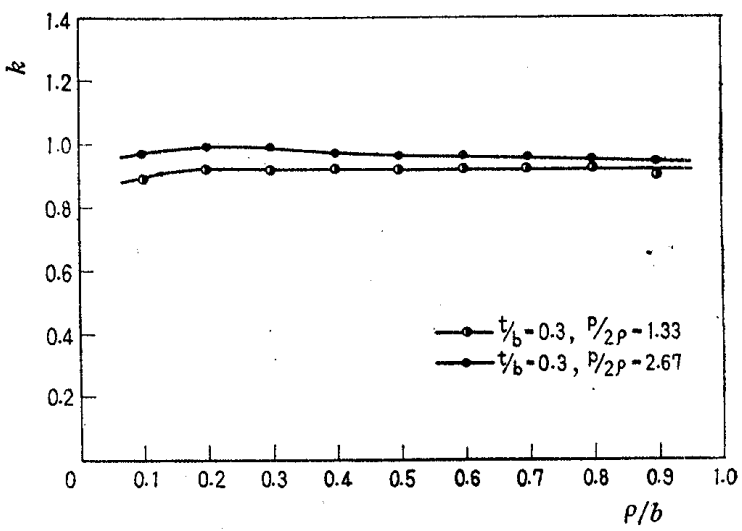

Fig. 15 Effect of the root radius on the $k$-values

Table 5 Results of experiment ( $k$-values) 


$$
0.1 \leqq t / b \leqq 0.9(1.33 \leqq p / 2 \rho \leqq 2.67)
$$

$0.0146(t / b)^{2}+0.1508(t / b)+0.8188$

$+\left[0.05597(t / b)^{2}-0.1171(t / b)+0.07014\right] \cdot p / 2 o$

Lastly, Fig. 15 shows the $k$-curves for the effect of the root radius, corresponding to Fig. 11 ; $k$-curves indicate different shapes from the $\alpha$-curves in Fig. 11 , showing gentle peaks at about $\rho / b=0.2$ in both cases. In this case also, $k$ takes a value less than unity for the whole range of $\rho / b$. The experimental formulas for this case are as follows:

$$
\begin{aligned}
& 0.1 \leqq \rho / b \leqq 0.9 \quad(1.33 \leqq p / 2 \rho \leqq 2.67) \\
& -0.2173(\rho / b)^{2}+0.3048(\rho / b)+0.7687 \\
& \quad+\left[0.0694(\rho / b)^{2}-0.1164(\rho / b)+0.0806\right] \cdot p / 2 \rho
\end{aligned}
$$

As is seen from the above experimental results, the value of $k$ is less than unity, in other words, $\alpha_{2}$ is smaller than $\alpha_{1}$, except for larger values of $p / 2 \rho$ in the case of the effect of the pitch. This result is assumed to be valid for other shapes of notch than the $U$ notch studied in this experiment. Accordingly, the stress concentration factor could be reduced by forming a supplementary notch of the same shape (this notch may be called a stress concentration reduction notch) in the neighbourhood of the master notch. This fact is noteworthy in the design of notched members.

\section{Conclusions}

From the experimental results above-mentioned, the following conclusions may be drawn.

(1) As to the effect of the pitch, the stress concentration factor $\alpha_{2}$ of two notches is less than that $\alpha_{1}$ of a single notch for small values of $p / 2 \rho$ owing to the overlapping effect, reaching a minimum at a certain value of $p / 2 \rho$. For larger values of $p / 2 \rho$ than this critical value, $\alpha_{2}$ increases inversely, approaching finally to $\alpha_{1}$.

(2) The stress concentration factor of an elongated notch which is formed by cutting off the intermediate part between two notches shows a smaller value than $\alpha_{2}$. This fact is of importance in the design of notched members.

(3) With respect to the effect of the notch depth, $\alpha_{2}$ increases steeply for small values of $\rho / b$, reaching a peak, and thereafter decreases gradually to unity at $t / b=1.0$. This tendency is quite similar to that of a single notch, but $\alpha_{2}$ is less than $\alpha_{1}$ for the whole range of $t / b$ between zero and unity. Moreover, the peak of $\alpha_{2}$ occurs at larger values of $p / 2 \rho$ for a deeper notch.

(4) With regard to the effect of the root radius, $\alpha_{2}$ diminishes steeply for small values of $\rho / b$, and thereafter slowly, which is also a similar trend to the case of a single notch. Again, $\alpha_{2}$ is less than $\alpha_{1}$, and this is especially remarkable for a smaller value of $p / 2 o$.

(5) The point at which the maximum fringe order appears on the notch contour shifts slightly to the outside of the notch root for certain ranges of $p / 2 \rho, t / b$ and a whole range of $\rho / b$, producing a deviation angle $\theta$. The maximum value of $\theta$ was $5^{\circ}$ to $8^{\circ}$ in this experiment.

(6) To obtain some relation between $\alpha_{2}$ and $\alpha_{1}$, we put these in the formula $\alpha_{2}=k \cdot \alpha_{1}$ (this coefficient $k$ may be designated a stress concentration reduction factor). The values of $k$ calculated from the experimental results were less than unity in nearly all cases. In other words, the overlapping effect of two notches appeared in the whole field of this experiment except a certain case of the effect of the pitch. This offers a possibility of reducing the stress concentration of a single notch by forming a supplementary notch of the same shape in its neighbourhood, and is a noticeable fact for the designers. From the experimental results, $k$-curves were drawn for each case, and experimental formulas were established.

\section{Acknowledgements}

The authors wish to express gratitude to $\mathrm{Mr}$. H. Kurozawa and Mr. T. Hayashida for their help and assistance in this experiment.

\section{References}

(1) Y. Murakami and T. Kawabe: Trans. Japan Soc. Mech. Engrs., Vol. 26, No. 161 (1960), p. 57; Vol. 26, No. 170 (1960), p. 1387.

(2) M.Nishida: Bulletin of the Scientific Research Insi., Vol. 3 (1943), p. 62; Vol. 4 (1943), p. 97.

(3) H. Neuber: Kerbspannungslehre, (1958), S. 161, Springer-Verlag.

(4) F. Hirano: Trans. Japan Soc. Mech. Engrs., Vol. 16. No. 55, (1950), p. 52; Vo1. 17, No. 61 (1951), p.

(5) A.J. Durelli et al : Proc. Soc. Expr. Stress Analysis, Vol. 10, No. 1 (1952), p. 53.

(6) H. Okubo: Jour. Japan Soc. Mech. Engrs., Vol. 62, No. 488 (1959), p. 1272.

(7) Y. Murakami and T. Kawabe : Jour. Japan Soc. Test. Materials, Vol. 11, No. 100 (1962), p. 13.

(8) R.E.Peterson : Stress Concentration Design Factors, (1953), p. 69 . 